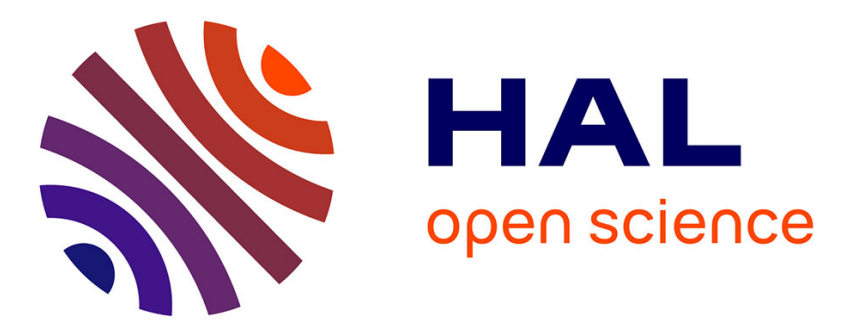

\title{
Robust time-frequency model estimation in otolith images for fish age and growth analysis
}

\author{
Ronan Fablet, Abdesslam Benzinou, Christian Doncarli
}

\section{To cite this version:}

Ronan Fablet, Abdesslam Benzinou, Christian Doncarli. Robust time-frequency model estimation in otolith images for fish age and growth analysis. ICIP 2003 : IEEE International Conference on Image Processing, Sep 2003, Barcelone, Spain. pp.593 - 596, 10.1109/ICIP.2003.1247314 . hal-02283315

\section{HAL Id: hal-02283315 https://hal.science/hal-02283315}

Submitted on 10 Sep 2019

HAL is a multi-disciplinary open access archive for the deposit and dissemination of scientific research documents, whether they are published or not. The documents may come from teaching and research institutions in France or abroad, or from public or private research centers.
L'archive ouverte pluridisciplinaire HAL, est destinée au dépôt et à la diffusion de documents scientifiques de niveau recherche, publiés ou non, émanant des établissements d'enseignement et de recherche français ou étrangers, des laboratoires publics ou privés. 


\title{
ROBUST TIME-FREQUENCY MODEL ESTIMATION IN OTOLITH IMAGES FOR FISH AGE AND GROWTH ANALYSIS
}

\author{
Ronan Fablet ${ }^{1}$, Abdeslam Benzinou ${ }^{2}$ and Christian Doncarli ${ }^{3}$ \\ ${ }^{1}$ IFREMER/LASAA \\ BP 70, 29280 Plouzane,France \\ rfablet@ifremer.fr \\ ${ }^{2}$ ENIB/RESO \\ BP 30815, 29280 Plouzane,France \\ benzinou@enib.fr \\ ${ }^{3} \mathrm{ECN} / \mathrm{IRCCyN}$ \\ BP 9210144321 Nantes,France \\ Christian.Doncarli@irccyn.ec-nantes.fr
}

\begin{abstract}
We present a robust method for time-frequency model estimation. It involves a robust Leclerc's estimator to ensure robustness w.r.t. noise and interferences present in timefrequency representations. This scheme is applied to fish age and growth analysis from otolith images. This application involves the estimation of the parameters of a priori fish growth models using this robust time-frequency analysis. We present a quantitative experimental validation over a large set of real images of Plaice otoliths.
\end{abstract}

\section{PROBLEM STATEMENT}

Fish age and growth estimation is of key importance for marine living resources assessment and ecology applications. It mainly relies on the analysis of calcified structures, such as fish otoliths. Otolith images, as depicted by Fig.1 for a Plaice, are composed of successive concentric dark and light rings. Fish age is determined by counting these rings, whereas fish growth analysis consists in measuring the evolution of the distances between the successive rings. These tasks are routinely achieved by human readers, but they are extremely tedious for large sets of otoliths (typically, several thousands of otoliths a year) and depend on reader's subjectivity.

Different studies have already investigated computer vision techniques to develop automatic or semi-automatic tools for fish ageing issues from otolith images [3, 4]. Mainly, proposed approaches exploit either peak and valley detection on 1D radial signals taken from the nucleus to the edge of the otolith [4], or 2D techniques (such as deformable models [3]) exploiting ring continuity. In both cases, a knowledge of the fish otolith growth pattern would greatly ease ring detection, as highlighted by [4] using a priori growth models.

In this paper, we propose a framework for the direct estimation of the otolith growth pattern from a two-dimensional time-frequency analysis [6] of 1D otolith image radials. More Precisely, given an image otolith such as in Fig.1.a, we extract a radial from the nucleus to the edge of the otolith. As

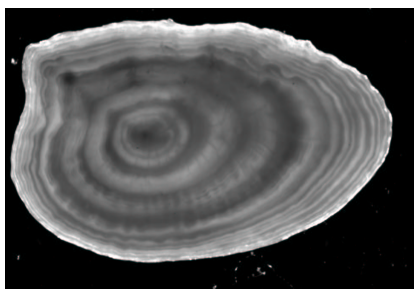

a

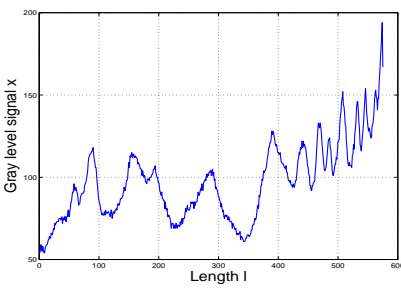

b
Fig. 1. Example of a Plaice otolith: a) image of a Plaice otolith annual rings present on a plaice otolith displaying seasonal white and dark rings, b) gray level plot along an image radial taken from the nucleus to the edge of the Plaice otolith.

displayed in Fig.1.b, the resulting gray level 1D signal involves oscillations corresponding to the successive growth rings. In fact, the growth pattern can be viewed as the frequency modulation of this real biological signal. Growth demodulation will then lead to a simple periodic signal, the period of which is given by the temporal period of ring appearance (e.g., one year in our case for the Plaice). Timefrequency analysis [6] is well-suited to process this category of non-stationary signal. Given a 1D time signal, it basically results in a two-dimensional time-frequency representation of signal content, which indicates signal frequencies at each time instant. Based on a priori growth modeling, we directly achieve the estimation of the parameters of the growth model from the two-dimensional time-frequency weight map within a robust framework. Robust estimation [7] is of key importance in our case due to to the low quality of the computed time-frequency representation, as highlighted by Fig. 2 .

This paper is organized as follows. Section 2 presents the use of time-frequency analysis for growth demodulation. We present the framework for robust growth model estimation in the time-frequency representation in Section 3. Section 4 presents the experimental evaluation of our approach on a set of real Plaice otolith images, and concluding remarks are given in Section 5. 


\section{GROWTH MODULATION AND TIME FREQUENCY ANALYSIS}

\subsection{Growth modulation}

Given an otolith image, we extract a radial from the nucleus to the edge of the otolith. The resulting 1D signal represents the evolution of the gray level as a function of the distance along the considered radial, as illustrated by Fig.1. We denote this signal $y(l)$, where $l$ is the length computed from the nucleus taken within the interval $[0, L]$ with $L$ the radial length. As in [4], this radial gray level profile is extracted in a robust manner to reduce the noise level. In addition, to get rid of the long-term tendency due to lighting conditions, we apply a low-pass filter. Let us denote by $x(l)$ the resulting signal.

Since the biological ring signal underlying $x$ is known to be one-year periodic, $x$ is expressed as the result of the growth modulation $\Phi(l)$ :

$$
x(t)=A \sin (2 \pi t)=A \sin (2 \pi \Phi(l))
$$

where time instant $t$ is simply related to growth modulation by: $t=\Phi(l)$. Our goal is then to determine this growth modulation, from which we will directly deduce the growth pattern $l=\Phi^{-1}(t)$.

\subsection{Time-frequency representations}

Due to the non-stationarity of the processed signal $x$, Fourier analysis will only allow to evaluate its frequency content, but not to track these frequency components w.r.t. length $l$. On the contrary, bilinear transforms used for time-frequency analysis [6] provide a well-suited tool to visualize both signal frequency components and their temporal localization from a two-dimensional time-frequency weight map. We will focus on the transforms of Cohen's class.

The reader should note that length variable $l$ plays a role similar to time $t$ in more standard signal applications, whereas the time notion here refers to the one-year periodic biological signal. Thus, the time-frequency analysis we will achieve should be viewed as a length-spatial frequency analysis. We will in particular use the Wigner-Ville transform, from which all bilinear transforms of Cohen's group can be deduced. For given length $l$ and frequency $f$, the coefficient $w v(l, f)$ of the Wigner-Ville transform is given by:

$$
w v(l, f)=\int x(l+\tau / 2) x^{*}(l-\tau / 2) \exp (-j 2 \pi f \tau) d \tau
$$

The higher $w v(l, f)$, the more present frequency $f$ in signal $x$ at length $l$. In practice, we first apply the Hilbert transform to $x$ to reduce interference terms in the time-frequency representation [6]. Fig.2 displays the Wigner-Ville representation $w v$ for the signal depicted in Fig.1.

\section{ROBUST MODEL ESTIMATION}

In this two-dimensional time-frequency domain, the estimation of the modulation function $\Phi$ comes to determine the instantaneous frequency law $[1,2,6]$. Mainly, two categories of approaches can be considered: parametric and non-parametric techniques. The latter achieve the estimation of the instantaneous frequency law from peaks of the time-frequency representation, weighted average in the timefrequency domain [2], or hybrid approaches based on signal projection on a function basis [1]. However, as highlighted by the example of time-frequency representation given in Fig. 2 for a real otolith signal, we need to cope with noisy representation. This prevents from using a non-parametric scheme, as shown in the experiments.

On the other hand, parametric methods rely on a priori models of the instantaneous frequency law, for instance linear or hyperbolic models. The issue then comes to estimate the parameters of these a priori models. One can apply direct methods comparing the observed signal and the signal generated from estimated parameter. It seems however beneficial to take advantage of the two-dimensional timefrequency representation. This could be done in two stages involving first a non-parametric estimation of the instantaneous frequency law, and then a standard 1D model regression. Alternatively, we propose a technique exploiting the whole time-frequency representation to achieve model parameter estimation in the time-frequency domain within a robust framework.

\subsection{A priori fish Growth models}

Different biological studies highlighted that fish growth patterns $l=\Phi^{-1}(t)$ are well described by a priori models. The most widely used one is Von Bertalanffy's model [4]. It leads to the following expression of the growth modulation function $t=\Phi(l)$ :

$$
t=\Phi_{\left(K, L_{\infty}\right)}(l)=-K \ln \left(L_{\infty}-l\right),
$$

where $K$ describes the slope of the growth curve, and $L_{\infty}$ the theoretical fish length at time $t=\infty$.

The instantaneous frequency $\phi(l)$ is given by the first order derivative of the growth modulation function: $\phi(l)=$ $d \Phi(l) / d l$. The a priori model to be considered in the timefrequency domain is then:

$$
f=\phi_{\left(K, L_{\infty}\right)}(l)=K /\left(L_{\infty}-l\right)
$$

\subsection{Robust parameter estimation}

Given a signal $x$, we compute the associated time-frequency representation $w v(l, f)$. The estimation of the parameters $\Theta=\left(K, L_{\infty}\right)$ of the a priori model is stated as the maximization of the expectation $E_{\Theta}(w v)$ of the time-frequency 
function $w v$ w.r.t. a Gaussian kernel with variance $\sigma$. It comes to:

$$
\begin{gathered}
\widehat{\Theta}=\arg \max _{\Theta} E_{\Theta}(t f r) \\
\widehat{\Theta}=\arg \max _{\Theta} \int t f r(l, f) \frac{1}{\sqrt{2 \pi \sigma^{2}}} \exp -\frac{[f-\phi(l)]^{2}}{\sigma^{2}} d l d f
\end{gathered}
$$

In practice, we exploit a discrete representation of the timefrequency plane $(l, f)$, such that $l \in\{0,1, . ., L\}$ and $f \in$ $\left\{0, \Delta f, 2 \Delta f, \ldots, f_{\max }\right\}$, where $\Delta f$ and $f_{\max }$ are deduced from the choice of the bilinear transform, and the length $L$ of the processed radial. We finally need to solve for:

$$
\widehat{\Theta}=\arg \max _{\Theta} \sum_{l=0}^{L} \sum_{f=0}^{f_{\max }} \frac{t f r(l, f)}{\sqrt{2 \pi \sigma^{2}}} \exp -\frac{[f-\phi(l)]^{2}}{\sigma^{2}}
$$

This equivalently leads to the estimation of model parameters $\Theta$ in a robust minimization framework w.r.t. Leclerc's robust estimator $\rho(r)=\left[1-\exp -(r / \sigma)^{2}\right]$ [5], given the weights $w v$ in the time-frequency domain.

Robust statistics are widely used in computer vision and signal processing to cope with model estimation in noisy environment. These estimators enable to reject spurious data (outliers) to achieve an accurate estimation. Variance parameter $\sigma$ controls the amount of points rejected as outliers. Robust estimation is equivalently implemented as successive weighted least-square steps. At iteration $n$, given the current model parameter estimate $\Theta^{n-1}$, we first compute the robust weights $w(l, f)$ defined by: $w^{n}(l, f)=$ $\psi\left(f-\phi_{\Theta}^{n-1}(l)\right)$, where $\psi(r)=\rho^{\prime}(r) / r$ is the influence function of the considered robust estimator. Estimate $\Theta^{n}$ is then the solution of the following weighted least-square minimization:

$$
\widehat{\Theta}=\arg \min _{\Theta} \sum_{l=0}^{l=L} \sum_{f=0}^{f_{\max }} w^{n}(l, f) t f r(l, f)\left\|f-\phi_{\Theta}(l)\right\|^{2}
$$

Since $\phi_{\Theta}$ is not a linear w.r.t. $\Theta$, this minimization has no close-form solution. Hence, we consider an incremental strategy exploiting a linearized version of this quadratic criterion around the current parameter estimate $\Theta^{n}$.

Variance parameter $\sigma$ controls the rejection of outliers to determine the current estimate. Initially, $\sigma$ is fixed at a high value to let the minimization focus on areas of interest in the time-frequency domain. While the estimate is refined, $\sigma$ is decreased geometrically up to a minimum value. Initial parameter value $\Theta^{0}$ were set to a mean growth model.

Let us point out that this scheme for robust time-frequency model estimation is of general interest, and not limited to the specific hyperbolic case considered in this paper for application purposes. Other a priori models, for instance polynomial or exponential models, could be exploited similarly.

\subsection{Fish age and growth estimation}

Given the parameter estimate $\widehat{\Theta}$, the instantaneous frequency law $\phi_{\widehat{\Theta}}$ is given by relation (4). Then, the frequency modulation is computed as: $t=\Phi_{\widehat{\Theta}}(l)=\int_{0}^{l} \phi_{\widehat{\Theta}}(s) d s$. The growth pattern $l=\Phi_{\widehat{\Theta}}^{-1}(t)$ is then numerically estimated from $\Phi_{\widehat{\Theta}}$, as well as fish age.

\section{EXPERIMENTS}

We have carried out experiments on a set of 116 Plaice otolith images. For each otolith image, one radial was manually extracted, and fish age and growth pattern were determined by a reader. This provides us with a ground truth to evaluate our approach. This set involves samples from age class 1 to 11 .

In Fig.2, we illustrate the different steps of our approach. We display the signal extracted from otolith image after a low-pass filtering to suppress long-term tendency. The Wigner-Ville time-frequency representation of this signal is also presented. Estimated instantaneous frequency laws for four approaches (a non-parametric peak-based approach (NPPBIF), the non-parametric method presented in [1] (NPHIF), a parametric approach using model regression from peakbased instantaneous frequency law (PBPIF) and the robust scheme presented in this paper (RPIF)). Obviously, this timefrequency representation is too complex to enable a relevant non-parametric processing. This also explains the better result obtained by the robust parametric technique (RPIF) compared to the parametric peak-based one (PBPIF). In the third subfigure of Fig.2.c, the growth patterns estimated using these two parametric techniques are compared to reader's ground truth. The robust approach supplies a more accurate estimation. Similarly, fish age is estimated to 11.2 by this technique and to 14.3 by the peak-based parametric approach, while reader's ground truth age is 11 . This example demonstrates the interest of our robust framework for image-based fish age and growth analysis.

In addition, we exploit the whole set of 116 Plaice otoliths to provide a quantitative evaluation of our approach. We present estimation results of fish age and growth analysis for two age groups: the first group includes fish aged from 0 to 6 , and the second from 7 to 11 . Fig. 3 presents these results in terms of estimation errors w.r.t. reader's ground truth, mean error, and error standard deviation. For the first age group, both techniques behave similarly but poorly. In fact, the number of growth rings (oscillations) is too small to use time-frequency analysis. For the second age group, the robust method provides relevant age and growth estimates and significantly outperforms the parametric peakbased technique. Besides, tests carried out with other timefrequency representations (Choi-Williams, spectrogram, reallocated spectrogram), brought no particular improvements. 

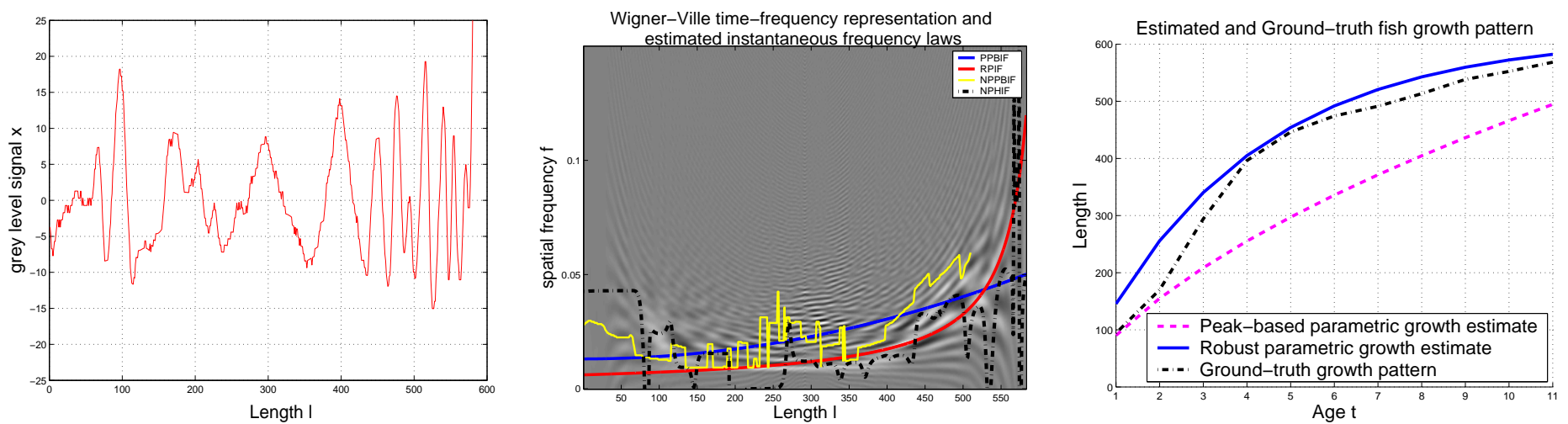

Fig. 2. Illustration of the different steps of the fish age and growth pattern estimation: a) low-pass filtered version of the signal depicted in Fig.1, b) Wigner-Ville time-frequency representation with superimposed estimated instantaneous frequency laws (see text for details), c) comparison of estimated growth patterns to reader's ground truth.

Fish age estimation error

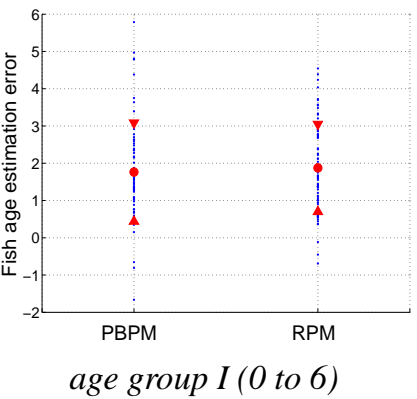

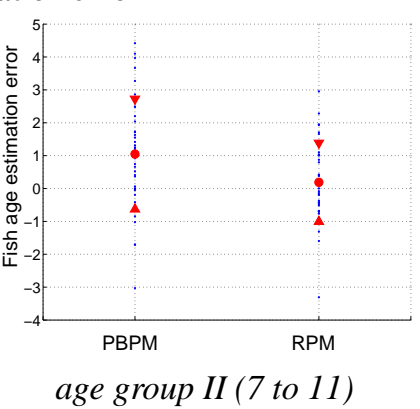

Fish growth estimation error

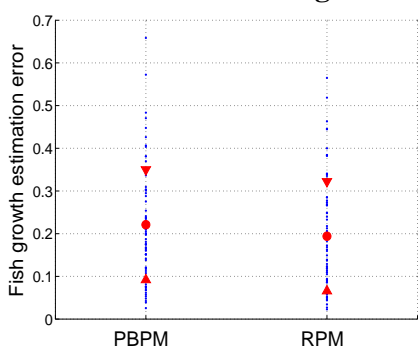

age group I (0 to 6)

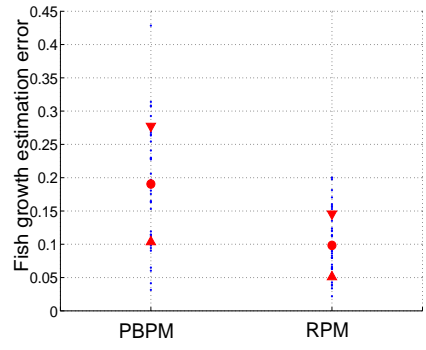

age group II (7 to 11$)$

Fig. 3. Results of fish age and growth pattern estimation for age class from 1 to 6 (age group I), and 7 to 11 (age group II) for the robust parametric method detailed in this paper, and a parametric peak-based approach. We display the estimation errors, their mean and their standard deviation.

\section{CONCLUSION}

We have presented in this paper a robust parametric technique for model parameter estimation in two-dimensional time-frequency representations. We performed a quantitative evaluation for an application to image-based fish age and growth pattern estimation, for which our approach favorably compares to a standard parametric peak-based method.

In future work, we plan to exploit the growth pattern estimation to enhance age estimation based on ring detection, either directly on the demodulated signal, or as an a priori to lead 2D ring detection such as deformable models [3]. In particular, 2D techniques are known to be less accurate for older ages, for which the proposed robust time-frequency analysis was shown to be relevant.

\section{Acknowledgements}

The authors are grateful to Auger etal. for the availability of the Matlab time-frequency toolbox and also wish to thank J. Le Bihan, head of RESO Laboratory at Ecole Nationale d'Ingnieurs de Brest, for his support and encouragement.

\section{REFERENCES}

[1] R.G. Baraniuk, M.J. Coates and P. Steeghs, "Hybrid linear/quadratic time-frequency attributes," in IEEE Trans. Signal Proc., vol. 49, no. 4, April 2001, pp. 760-766.

[2] B. Boashash and P. O'Sheah "Use of cross Wigner-Ville distribution for estimation of instantaneous frequency," in IEEE Trans. Signal Proc., vol. 41, no. 3, March 1993, pp. 1439-1445.

[3] A. Benzinou, H. Troadec, J. Le Bihan, V. Rodin, H. de Pontual, J. Tisseau, "The locally deformable B-bubble model : an application to growth ring detection on fish otoliths," in Proc. of Scand. Conf. on Image Analysis, SCIA'97, pages 181-187, Lappeenranta (Finland), June, 1997.

[4] F. Lagardre and H. Troadec, "Age estimation in common sole solea solea larvae: validation of daily increments and evaluation of a pattern recognition technique," in Marine Ecology Progress Series, 1997, vol. 155 , pp. 223-237.

[5] Y.G. Leclerc "Constructing simple stable descriptions for image partitioning," in Int. Jal on Computer Vision, 1989, vol. 3, no. 1, pp. 73-102.

[6] Patrick Flandrin, Time-Frequency/Time-Scale analysis, Academic Press, 1999.

[7] P. Huber, Robust Statistics, John Wiley \& Sons, 1981. 